\title{
CALCAREOUS NANNOFOSSILS (ODP 653) NONLINEAR COUPLINGS WITH MILANKOVITCH CYCLES: IMPLICATIONS FOR THE ORBITAL TUNING METHOD
}

\author{
CACHÃO, Mário, Dept. Geology, Univ. Lisbon, Rua Escola Politécnica, n 58, P-1294 LISBOA \\ CODEX, PORTUGAL
}

During the last decade the Milankovitch based orbital tuning method acquired an increasing importance as the stratigraphic framework for Pleistocene pelagic sections and/or as a tool to increase stratigraphic resolution on older marine sections. This cyclostratigraphic method has obvious advantages in relation with the traditional linear interpolation method, which generally uses only a few age-calibrated points and assumes that sedimentation rate was constant between those data. Because is quite difficult to validate this last assumption a certain relief arose with the possibility to replace this assumption by a more sophisticated one: that climate (generally expressed by its proxy $\delta$ ${ }^{18} \mathrm{O}$ ) is in phase with orbital induced changes is solar radiation that reaches Earth.

However, from models of orbital induced changes in ice volume during Pleistocene glacialinterglacial cycles some difficulties (delays, saw-tooth cyclicity, eccentricity's small effects) were derived on the particular aspects of how the Earth climate operates and ice caps may respond to orbital forcing. On the other hand, on shorter time scales, present day Climate has an intrinsic unpredictability, recently defined as chaotic behaviour. These were some of the reasons that led us to test, in a particular paleoceanographic scenario (Western Mediterranean), the Milankovitch assumption by using phytoplankton responses. We used phytoplanktonic entities since these photosynthetic organisms, theoretically, should also respond to changes in light intensity and so testify the presence and effect of Milankovitch cycle(s).

Our data was retrieved from the Pliocene section (4.66 to $1.85 \mathrm{Ma}$ ) of the ODP 653 (Leg 107), selected by its high recovery rate (main value $87.8 \%$ ) practically absent taphonomic bias and a well established biostratigraphic framework. Our time series were built by evaluation at optical petrographic microscope (x1250) of the percentage abundance of selected Calcareous nannofossils taxa. We also used three oxygen isotope time series from the same core retrieved from three distinct Planktonic Foraminifera taxa. All time series were equal time interpolated by rectangular integration and smoothed by a $5^{\circ}$ degree orthogonal polynomial function.

By performing spectral analysis (Simple Fourier Transform) we recognised red-shifted spectra signatures for the three above mentioned $\delta^{18} \mathrm{O}$ time series, which seems to suggest the influence of (paleo)environmental factors on the temporal behaviour of these proxies. After first differentiation (pre-whitening) to remove red noise, high variance peaks were obtained close enough to the eccentricity Milankovitch cycle ( $c a .100 \mathrm{ka}$ ) to allow us to interpret a possible relation. Nevertheless, if we tune the frequency of one of the $\delta^{18} \mathrm{O}$ time series to mach eccentricity the other two "signals" would still continue untuned. Which one would be chosen ?.

Performing the same Spectral Analysis on 16 Pliocene Calcareous nannofossils taxa we also obtained red-shift spectra. First differentiation was again applied to remove the "memory" of the system allowing to obtain quite distinct and sharp peaks for each one of the quantified taxa, also around the eccentricity frequency. Taking in consideration only the significative peaks and after their standardisation we could conclude that they were all centred exactly on the $95 \mathrm{ka}$ eccentricity cycle, defining a broad and symmetrical peak. It looked as if each taxa, although somehow influenced by the eccentricity orbital cycle (plus stochastic resonance ?) had its own frequency signal by some sort of vital effect mechanism (frequency-locking by large time scale ecological succession?).

In conclusion, using one of the $\delta^{18} \mathrm{O}$ time series to tune the age model to eccentricity would automatically untune the signal picked up by Coccolithophore and other related groups. So, it is essential to test our time series with a larger number of proxies ( $\delta^{18} \mathrm{O}^{\prime} \mathrm{s}$ and others) before deciding the need of tuning, using one or the "only one" $\delta^{18} \mathrm{O}$ time series available. Our data revealed to be possible to obtain several $\delta^{18} \mathrm{O}$ spectral signals for a single core interval, each one picking up a particular paleoenvironmental temperature-related factor and neither of them properly frequencylocked and in phase with orbital frequencies. What could be interpreted as sedimentary distortion may be an inherent property of natural (paleoclimatic/ paleoceanographic) systems. 